\title{
In-situ Bioremediation: Advantages of Parallel Computing and Graphical Investigating Techniques
}

\author{
M.C. Baracca, G. Clai, and P. Ornelli \\ ENEA HPCN Via Martiri di Montesole 4 40129-Bologna, ITALY \\ email: [baracca, ornelli] @bologna.enea.it
}

\begin{abstract}
The mathematical modelling and the simulation of the bioremediation process are fairly complex and very computational demanding. The use of parallel computers allows the prediction of the effects of bioremediation interventions safely and cheaply in a realistic timescale. Nevertheless, the data amount resulting from a real field simulation is so large that the only way to analyze it, in an effective way, is to work with its graphical interpretation, converting the huge numerical data volumes into $3 \mathrm{D}$ animation.
\end{abstract}

\section{Introduction}

The quality, duration and cost of a bioremediation intervention depend on the success of laboratory or pilot plant experiments, which are time consuming and not always reliable. Since the mathematical modelling and the simulation of the bioremediation process are fairly complex and very computational demanding, they can be used to predict in a realistic time-scale the effects of their actual application safely and cheaply, only through the exploitation of parallel computers.

Nevertheless, the data amount resulting from a real field simulation is so large that the only way to analyze it, in an effective way, is to work with its graphical interpretation, converting the huge numerical data volumes into 3D animation.

The two-years collaborative COLOMBO Project funded by the European Union has succesfully dealt with the application of both parallel computing and graphical investigating techniques to the simulation of the in situ bioremediation of contaminated soil.

\section{The Bioremediation Model}

The decontamination process works by stimulating the growth of indigenous bacteria and the model predicts the effects of bioremediation starting from geological, chemical and microbiological data as well as experimental results from the pilot plants designed and realized in the framework of the project. In order to solve succesfully soil pollution problems, researchers must simulate contaminant transport, diffusion and transformation processes and, at the same time, estimate the many parameters defining the bioremediation strategy.

The cellular automata (CA) modelling technique[1] was adopted in order to describe the phenomena taking place at the intermediate scale inside a portion of soil rather 
then the microscopic phenomena involved. The three dimensional bioremediation model[2], composed of mesoscopic size cells, describes real field interventions referred to heterogeneous regions simulating the fluid flow with diffusion and transport of pollutant inside the soil. The rule governing the system evolution has a "layered" structure, where the first layer is related to the water and pollutant flow through a porous saturated or unsaturated soil, the second describes the chemical behaviour and the third deals with the interaction between chemicals and biomass.

The model was tested on two real fields cases in Germany, the first at the US-Depot in Germersheim and the second near the Deutsche Bahn in Frankfurt.

\section{The Bioremediation Simulation Code}

The theory of CA lends itself well to the bioremediation simulation. In our case the automaton is related to a finite three-dimensional space and consists of a number of cells. Each of them is associates with a set of 6 neighbouring cells and its state is defined by a set of attributes, which are known as substates. The automaton evolves through a specified rule that decides the state of each cell in the next time-step based on the current state of the cell in question and those of its neighbours. The locality of the considered phenomena (bacteria and pollutants interact gradually over time and only within their neighbourhood area) benefits from parallel computers since one can decompose the automaton domain and map the components to cooperating computing processors with relative acceptable communication overhead, due to the boundary data exchange. Moreover, the CA approach is appropriate since it allows both to represent system heterogeneities, simply setting proper parameter values, and to tailor the cell sizes according to the problem needs and the available computing resources. The code has two modes and its execution is managed by a computational steering mechanism switching from the fluid dynamical to the biochemical layers simulation (and viceversa) when proper condition are satisfied. A real intervention simulation requires a very large number of iterations, it can run from several hours to some days, depending on whether soil conditions are saturated or unsaturated.

The benchmarks on CrayT3E-900 [3] and Beowulf cluster, have established that the code execution scales well with the number of available processors, as long as the size of the per processor data is more than half of the exchanged boundary data. In the pictures below, the benchmark results for the large model $(256 \times 128 \times 13$ cells) on the Cray platform, performed by the Edinburgh Parallel Computing Center researchers, are provided toghether with analogous benchmark results for the medium model (128x96x13 cells) on a Beowulf cluster. 


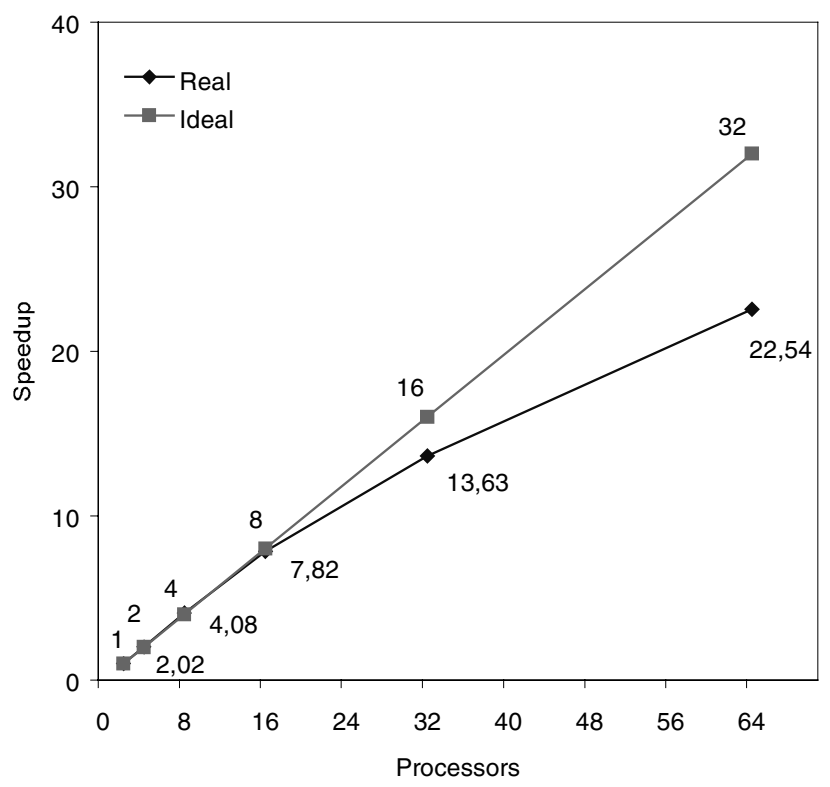

Fig. 1: Speedup (blue) and optimum speedup (red) scaling curves for the large model on the Cray T3E (2-64 processors)

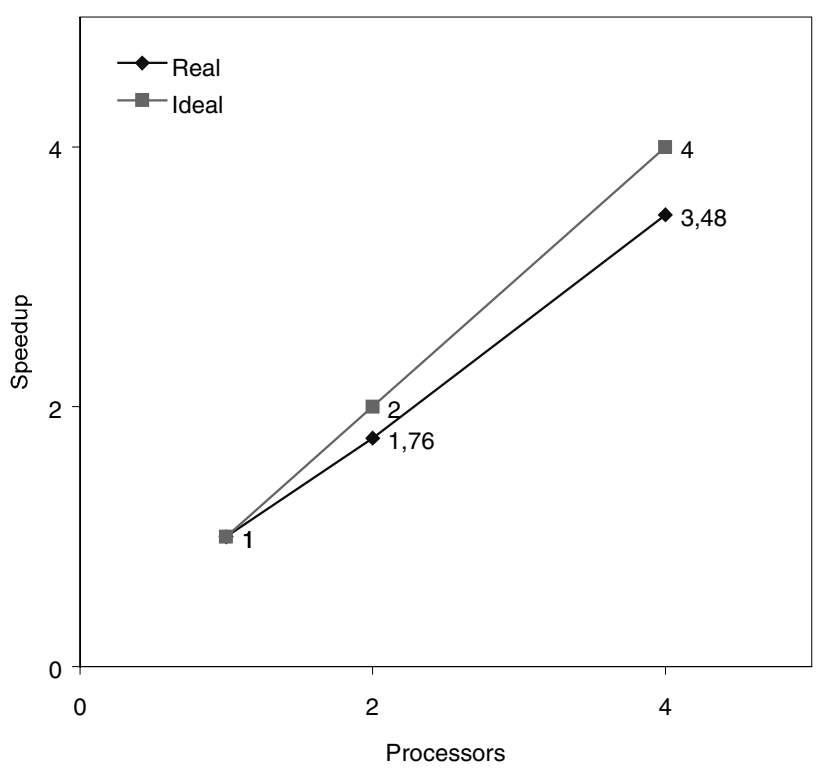

Fig. 2: Speedup (blue) and optimum speedup (red) scaling curves for the medium model on Beowulf cluster (1-4 processors) 


\section{The 3D-Visualization Application}

In the framework of the COLOMBO Project has been produced a user oriented interface[4], consisting in a graphical environment allowing to enter the data preprocessing and 3D visualization post-processing facilities as well as to run the batch simulation and the parameters optimization algorithms. This tool has been developed on an SGI Onyx2 machine using AVS/Express[5], a commercial visualization package, portable across several platforms of industrial interest. Then it has been ported succesfully on a Beowulf cluster and linked to the LessTif freeware libraries instead of the Motif libraries that were recommended by the manifacturer.

The remainder of this section is focused on the postprocessing application devoted to the graphical investigation of the bioremediation simulation outcome.

\subsection{The Postprocessing Investigation}

There are two main reasons of using visualization for numerical simulations: analyzing results and presenting them. A bioremediation intervention simulation is fairly complex and it can take great advantage from sophisticated 3D visualization, since the dynamics of different physical and chemical processes are better observed in graphical form. Starting from raw data gathered during the simulation, the researcher may analyze, study and discover useful features and numerical results that cannot be appreciated during the simulation. The interplay between the visualization and the simulation software, besides constant monitoring of intermediate states, enables the user to adjust on the fly the bioremediation intervention strategy, without the complete re-execution of the simulation.

On the other hand, given that COLOMBO is an industrial project, the presentation in graphical form of the bioremediation process predictions is crucial for proving the advantages and the reliability of the proposed strategy.

\subsection{The Visualization Features}

The methods of visualization are very important for the analysis of the numerical results and their presentation. They should be chosen accordingly to the phenomena in object, in order to give evidence to their more relevant features and more interesting aspects, and to allow the researcher to delve into them. In the present case of the bioremediation intervention model, each substate is interpolated and visualized by means of orthoslices, isosurfaces and volumes in a three dimensional Cartesian space representing the soil portion in terms of cells. The user can activate or deactivate each of the geometrical visualization modalities and the implemented features. Moreover, it is possible to rotate, to shift and to zoom as desired the 3-D picture in the visualization space.

Each orthoslice module subsets a cells array by extracting one slice plane perpendicular to one of the Cartesian axis. The user can move interactively the orthoslices in the range from 0 to the axis maximum dimension. When using this modality, with a mouse click, it is possible to retrieve the substate value and the 
coordinates of a cell by means of the probe feature, cutting out the interpolated data produced by the visualization process.



Fig. 3

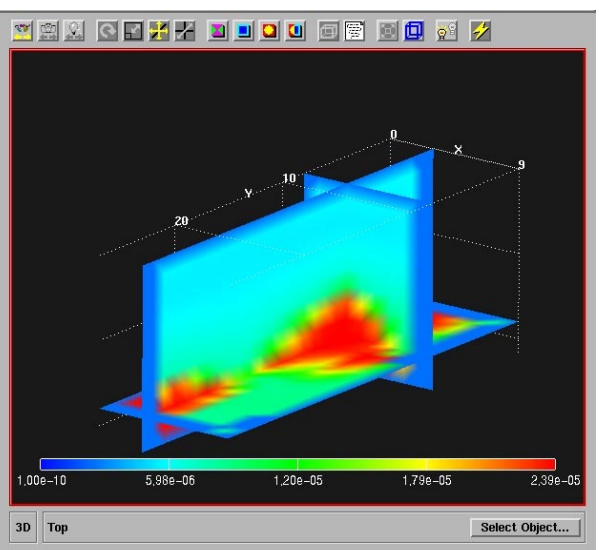

Fig. 4

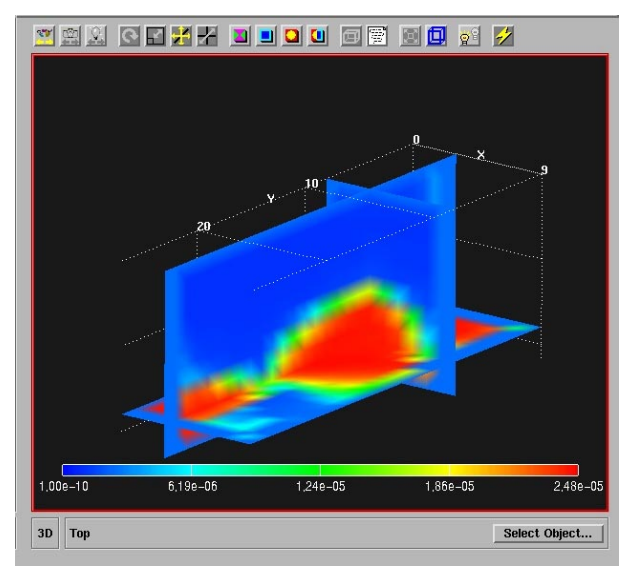

Fig. 5

Figures 3, 4, 5 show the 3D automaton, representing the Frankfurt real field test case. In this example, the visualization is performed by means of orthoslices. Specifically, the bacteria able to degrade the contaminants are investigated: the pictures sequence 
shows three time steps of the biomass growth, starting from the beginning of the remediation intervention.

In order to outline correlated substates or threshold effects, the isosurface module, that creates a surface with a given constant value level, has been improved including the option to map on a substate isosurface the cell values of an other substate.

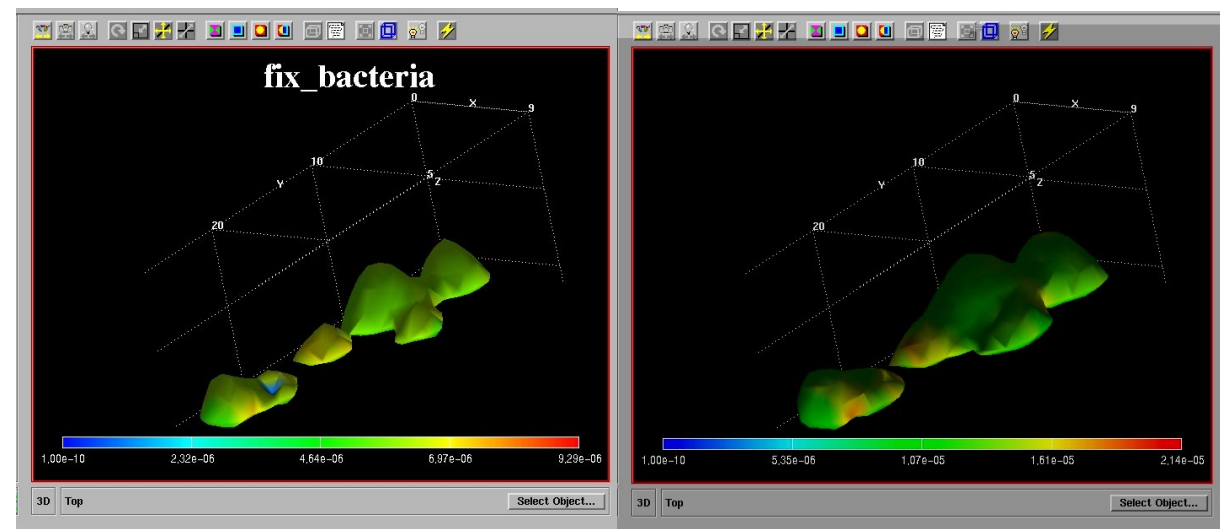

Fig. 6

Fig. 7

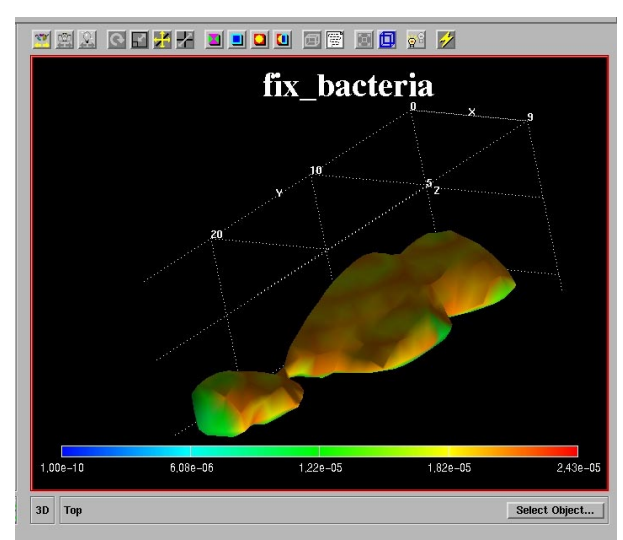

Fig. 8

In figures $6,7,8$, the 3D automaton, representing the Frankfurt real field test case, is visualized by means of the isosurface feature. This example shows two correlated 
substates, the oxigen concetration in water and the biomass. Given a specific value of the oxigen concentration, the related isosurface shape is created while the corresponding bacteria distribution is mapped onto it. The color coding scheme is presented at the bottom of each window. The pictures show three subsequent time steps of the biomass growth corresponding to the same oxigen concentration level.

The isovolume module that displays the interpolated volume of the cells whose values are greater or less than a specified level, as well as the bounded volume, displaying the substate volume included beetween two chosen cell values, are the bases for quantitative and statistical evaluations.

Vectorial fields visualization has been provided, in order to show in an effective way the air, water and pollutant fluxes. The vector fields are visualised by arrows, each one oriented as the local flux direction; the arrow color, mapped within a suitable color scale, represents the local flux value. Vector field planes can be visualized one at a time: the user can change the orthogonal plane and move it along the selected direction while the visualization is being performed.

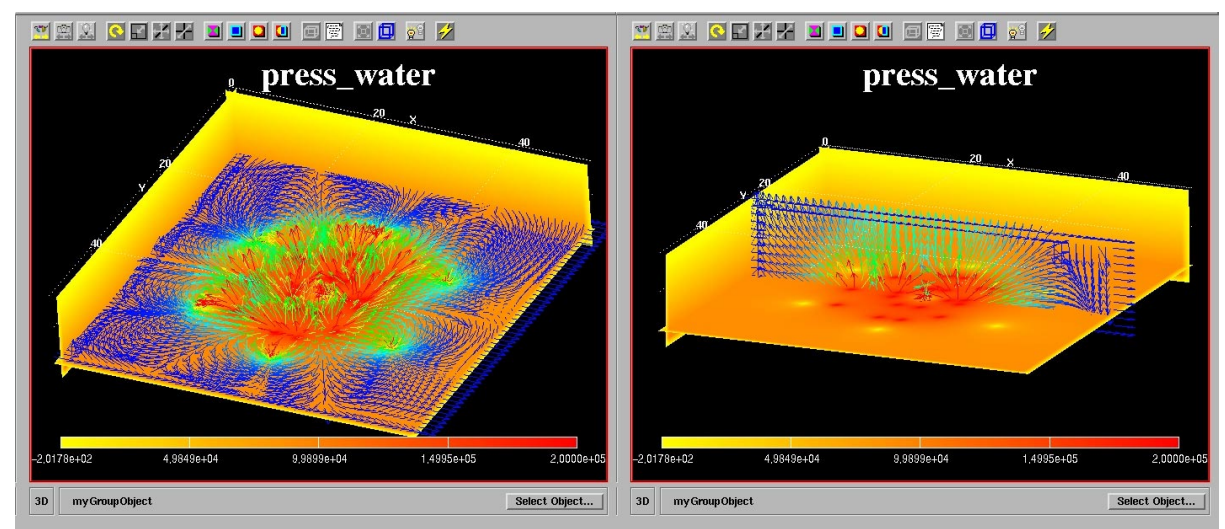

Fig. 9

Fig. 8

Figures 9 and 10 refer to the real field test case located in Germersheim. In the pictures the water flux is analyzed, shifting the attention from an horizontal to a vertical flux plane superposed to the water pressure distribution, at the injection and extraction wells level.

From the analysis of the bioremediation model applied to real field emerged that, usually, the surface dimensions are much larger than the field depth, so that the automaton cells look like a parallelepiped and not a cube. In order to properly visualize the automaton in this case, the Scale facility allows to choose and set a different scale for each axis. The Scale factor affects at the same time the scalar 
substate as well as the vector fields, so that in case of a superposition of a substate and a vector field, a change in the Scale factor results in a rescaling of the whole image.

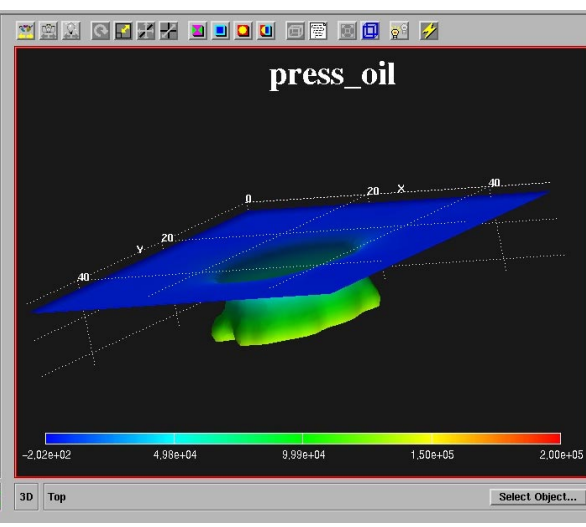

Fig. 9

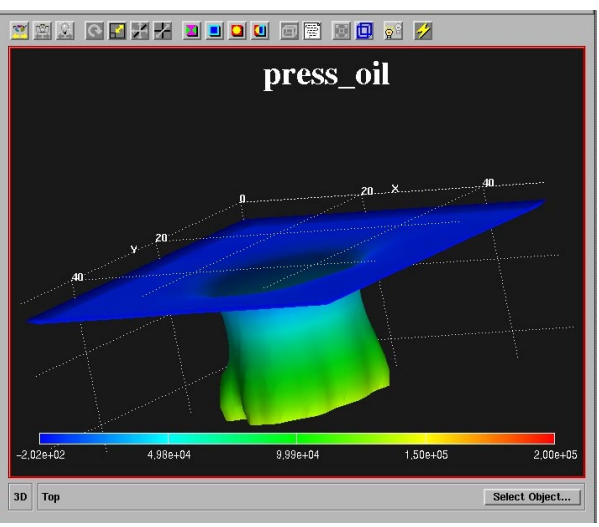

Fig. 10

In the figures 11 and 12, the Scale facility is applied to the contaminant pressure distribution mapped on a potential isosurface.

Since the dynamics of different physical and chemical processes are better observed in graphical form, the capability to produce 3D Animation visualizing the evolution of a bioremediation process, carried on according to a chosen intervention strategy, is crucial for demonstrating the reliability of the postprocessing visualization as a powerful tool for planning and designing actual interventions. Therefore, the temporal evolution of the automaton substates, based on a sequence of data files saved during the simulation, has been provided and it can be performed according to each one of the implemented geometrical modalities, as well as for vectorial fields.

\section{Conclusions}

The bioremediation model reliability in evaluating the degradation rates, the code scalability allowing the prediction of the interventions duration in a realistic timescale and the advantages of the visualization tool have determined the success of the COLOMBO Project.

The 3D animation capability to present the interventions details and the remediation processes evolution, in an effective way, was essential for the end-user to win support of the Italian Ministry for Scientific Research for the study and the application of new bioremediation techniques to polluted sites located in Italy. 


\section{REFERENCES}

1. J.von Neumann, "Theory of Self Reproducing Automata", Univ.Illinois Press, Champaign, Ill.,1966.

2. M.Villani, M.Mazzanti, R.Serra, M.Andretta, S.Di Gregorio, "Simulation model implementation description", Deliverable D11 of COLOMBO Project, July 1999.

3. K.Kavoussanakis et al., "CAMELot Implementation and User Guide", Deliverable D9 of COLOMBO Project, September 2000.

4. M.C.Baracca, G.Clai, P. Ornelli, "Pre/Post Processor Description", Deliverable D12 of COLOMBO Project, July 1998.

5. Advanced Visual System Inc., "Using AVS/Express", July 1998. 